\title{
EL IMPACTO DE LA PANDEMIA DEL COVID-19 EN LAS ELECCIONES: ESPECIAL REFERENCIA AL CASO AUTONÓMICO ESPAÑOL
}

\author{
Carlos Fernández Esquer \\ Investigador García-Pelayo,
} Centro de Estudios Políticos y Constitucionales

\begin{abstract}
Cómo citar este artículo / Citation: Fernández Esquer, Carlos. (2021). El impacto de la pandemia del covid-19 en las elecciones: especial referencia al caso autonómico español.
\end{abstract}

Palacios Romeo, F. y Cebrián Zazurca, E. (coords.)

Elección y representación: una conjunción compleja. Perspectivas y problemas de los regímenes electorales en España, Colección Obras colectivas, Fundación Manuel Giménez Abad, Zaragoza.

DOI: https://doi.org/10.47919/FMGA.OC21.0307

SUMARIO: I. PLANTEAMIENTO: LAS ELECCIONES EN TIEMPOS DE PANDEMIA - II. LOS APLAZAMIENTOS DE LAS ELECCIONES MOTIVADOS POR LA PANDEMIA Y LOS PROBLEMAS JURÍDICOS QUE SUSCITARON EN GALICIA Y PAÍS VASCO - III. LAS MEDIDAS EXCEPCIONALES ADOPTADAS PARA LA CELEBRACIÓN DE LAS ELECCIONES DURANTE LA PANDEMIA - IV. UNA NUEVA AGENDA DE INVESTIGACIÓN - V. BIBLIOGRAFÍA

\section{PLANTEAMIENTO: LAS ELECCIONES EN TIEMPOS DE PANDEMIA}

La propagación mundial del covid-19 ha impactado con fuerza en la salud y el bienestar de personas de todo el mundo y ha trastocado múltiples aspectos de nuestra vida social, económica y política. Las actuaciones para combatir la pandemia están incidiendo de forma significativa en el normal funcionamiento de las instituciones y servicios públicos y, en muchos casos, están afectando al disfrute y ejercicio de los derechos fundamentales por parte de los ciudadanos. Uno de esos ámbitos ha sido sin duda el de las elecciones: la pandemia ha 
supuesto -y continúa suponiendo- un enorme desafío para la celebración de elecciones en todo el mundo y está obligando a las autoridades a repensar la forma en que deben organizarse los comicios durante este tipo de situaciones.

Se trata de una cuestión de indudable interés, pues, aunque las "elecciones" son un área de estudio que ha sido profusamente analizada desde las disciplinas del Derecho Constitucional y, sobre todo, de la Ciencia Política, el fenómeno de la suspensión o aplazamiento de las elecciones, o aquellas otras que se desarrollan en situaciones extraordinarias, han recibido hasta el momento una escasa atención académica. La pandemia del covid-19 es el suceso más relevante que ha afectado a la celebración y organización de elecciones a nivel internacional en las últimas décadas, pero no hay que olvidar que existe un abanico más amplio de posibles situaciones de emergencia. No solo hablamos de epidemias, sino de otro tipo de imponderables tales como desastres naturales (inundaciones, terremotos, huracanes, etc.) o conflictos armados y guerras civiles, en los que todo lo relativo a organización de las elecciones debe reajustarse tanto por razones humanitarias como para mantener unos estándares democráticos y de integridad en el proceso electoral aceptables (James, 2020a; James y Alihodzic, 2020). Sin embargo, apenas se ha reflexionado sobre la pertinencia e implicaciones de retrasar las elecciones en situaciones excepcionales o qué fórmulas jurídicas son las adecuadas para acordar una postergación electoral. $Y$, en aquellos casos en los que se decide mantener la fecha de las elecciones, cuáles son las medidas más apropiadas para garantizar el correcto desarrollo del proceso electoral y de las votaciones, o cuáles son las repercusiones de celebrar los comicios en estas circunstancias insólitas. Se trata, en definitiva, de asuntos insuficientemente explorados por la literatura del Derecho electoral y de la Política comparada, pero que resultan de enorme trascendencia para una correcta comprensión sobre el funcionamiento de las elecciones en nuestras democracias contemporáneas. $Y$ es que la forma en que las distintas autoridades resuelvan las problemáticas electorales en esta coyuntura extraordinaria será un buen termómetro para calibrar la salud de los Estados democráticos y de Derecho y de sus perspectivas en el medio plazo: las decisiones que se están adoptando, además de tener consecuencias inmediatas sobre la calidad de las democracias, sentarán importantes precedentes sobre el modo de organizar los procesos electorales que proyectarán sus efectos pro futuro. 
Hay que recordar que uno de los rasgos definitorios de los regímenes democráticos es precisamente la celebración regular de las elecciones, esto es, su carácter periódico, cuyo fundamento radica en la necesidad de renovar regularmente la legitimidad de quienes ejercen el poder en representación de los ciudadanos (Dahl, 1971). Es desde esta lógica desde la que debe leerse el artículo 21.3 de la Declaración Universal de los Derechos Humanos, el cual proclama que "la voluntad del pueblo es la base de la autoridad del poder público; esta voluntad se expresará mediante elecciones auténticas que habrán de celebrarse periódicamente, por sufragio universal e igual y por voto secreto u otro procedimiento equivalente que garantice la libertad del voto". También el apartado b) del artículo 25 del Pacto Internacional de Derechos Civiles y Políticos, el cual reconoce el derecho de los ciudadanos a "votar y ser elegidos en elecciones periódicas, auténticas, realizadas por sufragio universal e igual y por voto secreto que garantice la libre expresión de la voluntad de los electores". O, en fin, el artículo 3 del Protocolo I al Convenio Europeo de Derechos Humanos, precepto que se limita a expresar que los Estados parte "se comprometen a organizar, a intervalos razonables, elecciones libres con escrutinio secreto, en condiciones que garanticen la libre expresión de la opinión del pueblo en la elección del cuerpo legislativo". Sucede, sin embargo, que la expansión mundial del coronavirus en este año 2020 está dificultando sobremanera el respeto a la periodicidad de las elecciones en muchos lugares del mundo, debido a que el mantenimiento de los comicios en la fecha programada ordinariamente puede suponer una amenaza para la salud pública y, por extensión, a la propia integridad del proceso electoral.

Aunque no todos los países han declarado alguno de los estados de emergencia previstos en sus respectivas constituciones, sí lo han hecho muchos de ellos. Desde el punto de vista de los estándares internacionales, la Comisión de Venecia (2020), en su Compilación de opiniones e informes sobre estados de emergencia, advierte de que no hay ninguna regla formal que impida a los Estados celebrar elecciones 0 referéndums en tales circunstancias, aunque varias constituciones posibilitan -o incluso imponen- el retraso de los procesos electorales en caso de activarse alguno de esos estados. No en vano, "existe el riesgo de que los principios electorales fundamentales se vean socavados durante el estado de emergencia, en particular el principio de la igualdad de oportunidades"; y es asimismo "irrefutable que la derogación de los derechos civiles y políticos de las personas 
crea el riesgo de que los resultados no sean democráticos". Es decir, la Comisión de Venecia muestra su preocupación ante la posibilidad de que, en el transcurso de coyunturas excepcionales en las que se active el Derecho de excepción, los Estados no puedan asegurar el cumplimiento de los estándares internacionales y constitucionales en materia electoral, lo que, sumado a eventuales limitaciones de derechos derivadas de la declaración de estados de emergencia, podría comprometer el carácter democrático de los comicios (Sánchez Navarro, 2020: 332).

Así las cosas ¿cómo han reaccionado los distintos países ante la pandemia en lo que a las elecciones se refiere? En términos generales, encontramos dos grandes tipos de respuestas. Un importante grupo de países y regiones ha decidido suspender o posponer las elecciones. Mientras que otro grupo ha optado por mantener la fecha original de los comicios y seguir adelante con los preparativos, aunque adoptando medidas especiales para adaptarse a la situación y minimizar los riesgos. Estos dos tipos de respuestas entrañan implicaciones políticas y jurídicas de distinta índole y suscitan interrogantes también diferentes, por lo que serán examinadas por separado.

\section{LOS APLAZAMIENTOS DE LAS ELECCIONES MOTIVADOS POR LA PANDEMIA Y LOS PROBLEMAS JURÍDICOS QUE SUSCITARON EN GALICIA Y PAÍS VASCO}

En perspectiva comparada se advierte que una de las principales reacciones de las autoridades ante la posibilidad de que la votación pudiera exacerbar la expansión del covid-19 ha sido la de optar por el aplazamiento de sus elecciones. Según los datos ofrecidos por International IDEA (2020a), entre el 21 de febrero y el 13 de diciembre de 2020, al menos 75 países y territorios en todo el mundo han decidido posponer sus elecciones nacionales, subnacionales o la celebración de referéndums. Por mencionar algunos ejemplos, se han pospuesto las elecciones parlamentarias en Nueva Zelanda, Etiopía y Siria, las elecciones generales en Bolivia o Serbia, las elecciones presidenciales y legislativas en República Dominicana, las presidenciales en Polonia, la segunda vuelta de las elecciones locales en Francia, las locales en Rumanía o las elecciones autonómicas en Galicia y País Vasco. También se han aplazado referéndums relevantes, como el de Chile para determinar si la 
ciudadanía estaba de acuerdo con iniciar un proceso constituyente para dotarse de una nueva Constitución; el referéndum de reforma constitucional en Rusia destinado, entre otras cuestiones, a fortalecer la institución de la Presidencia; o el de Italia para decidir si procedía reducir el número de escaños en el Parlamento nacional.

¿Existe cobertura jurídica para posponer todos estos procesos electorales, ya sea suspendiéndolos indefinidamente hasta que las circunstancias permitan una nueva convocatoria, ya sea retrasándolos a una fecha concreta? En una primera exploración, encontramos constituciones que prevén la posibilidad de retrasar la convocatoria de elecciones en situaciones excepcionales o cuando se declara un estado de emergencia mediante la extensión del mandato del Parlamento o proscribiendo su disolución. Un ejemplo de esto último lo brinda la Constitución Española, cuyo artículo 116.5 preceptúa que "no podrá procederse a la disolución del Congreso mientras estén declarados algunos de los estados comprendidos en este artículo [alarma, excepción y sitio], quedando automáticamente convocadas las Cámaras si no estuvieren en periodo de sesiones".

Ahora bien, ni las constituciones ni la legislación electoral suelen contemplar la posibilidad de suspender elecciones ya convocadas, es decir, con procesos electorales ya iniciados, ante causas sobrevenidas que desaconsejan -o simplemente hacen inviable- la celebración de elecciones con las debidas garantías. Por ejemplo, la legislación electoral española solo prevé supuestos concretos que permiten, por causas de fuerza mayor, suspender el acto de votación en las mesas electorales el día de la jornada electoral (artículo 84.2 de la Ley Orgánica del Régimen Electoral General -LOREG-), o bien la suspensión del escrutinio provisional en las mesas electorales (artículo 95.2 LOREG). Además, se tipifica como delito electoral que los funcionarios públicos suspendan, sin causa justificada, cualquier acto electoral (artículo 139 LOREG). En definitiva, ni la legislación española ni otras de nuestro entorno europeo contemplan expresamente la posibilidad de suspender el conjunto del proceso electoral.

Conviene precisar que una cuestión relevante sobre la decisión de suspender o posponer las elecciones tiene que ver con que dicha decisión esté exenta de sospechas. Debe evitarse toda clase de recelo sobre la posibilidad de que los partidos en el gobierno estén manejando los tiempos del aplazamiento electoral 
en su propio beneficio, pues ello podría minar la confianza de los ciudadanos y del resto de fuerzas políticas sobre la neutralidad con la que se desarrolla el proceso electoral. No hay que descartar que gobiernos sin escrúpulos democráticos aduzcan la situación de emergencia para mermar los derechos de los candidatos opositores, intimidar a los medios de comunicación independientes o reprimir a organizaciones críticas, haciendo que las contiendas electorales no se desarrollen en un contexto de libertad e igualdad de oportunidades.

Pues bien, España ha sido precisamente uno de los países donde se han producido aplazamientos electorales durante estos meses. Como se ha mencionado, se vieron pospuestas las elecciones autonómicas en Galicia y País Vasco, previstas inicialmente para el 5 de abril de 2020, pero celebradas finalmente el 12 de julio de 2020. Es decir, se postergaron por un período algo superior a los tres meses. A pesar de la falta de cobertura constitucional y legal para efectuar una operación así, las mencionadas elecciones fueron efectivamente suspendidas por los respectivos decretos de los Presidentes gallego ${ }^{1}$ y vasco ${ }^{2}$, previa deliberación de los respectivos Consejos de Gobierno, oídos los partidos con representación parlamentaria (en País Vasco) o los grupos políticos más representativos (en Galicia), así como las respectivas juntas electorales autonómicas. Resulta relevante el hecho de que ambas exposiciones de motivos destacasen que la cancelación electoral se había producido previa consulta con los partidos políticos. No en vano, International IDEA (2020b), en sus recomendaciones sobre cómo actuar para preservar la legitimidad de unas elecciones, aconseja que la postergación de unas elecciones deba ser acordada de forma consensuada entre todos los principales partidos.

De manera similar a otros países, entre las razones que se adujeron destacan la imposibilidad de que la campaña electoral se desarrollase con las condiciones necesarias, evitar que se produjesen congregaciones de ciudadanos en los colegios electorales durante la jornada electoral, y evitar también que los electores hiciesen uso de urnas, cabinas, papeletas o sobres, debido al riesgo que ello representaba para la salud pública. También existían

\footnotetext{
${ }^{1}$ Decreto 45/2020, de 18 de marzo, del Presidente de la Xunta de Galicia, por el que se deja sin efecto la celebración de las elecciones al Parlamento de Galicia de 5 de abril de 2020.

${ }^{2}$ Decreto 7/2020, de 17 de marzo, del Lehendakari, por el que se deja sin efecto la celebración de las elecciones al Parlamento vasco del 5 de abril de 2020.
} 
problemas para la tramitación del voto por correo, tal como manifestó a los presidentes de las respectivas juntas electorales autonómicas, a través del presidente de la Junta Electoral Central, el Director de la Oficina del Censo Electoral. Así, apelando a principios generales de la legislación electoral, como los de soberanía popular, igualdad, pluralismo, transparencia, objetividad, impedimento del falseamiento de la voluntad popular y unidad de acto electoral, los decretos de ambos presidentes justificaron su capacidad para dejar sin efecto las convocatorias de elecciones (Cebrián, 2020).

Por lo demás, ambos decretos presidenciales estipulaban que la convocatoria de elecciones se activaría de nuevo una vez pudiesen garantizarse de manera efectiva dichos principios. Ahora bien, encontramos diferencias en los dos casos. Así, mientras que el artículo 2 del Decreto 7/2020 del Lehendakari preveía la activación de la convocatoria de elecciones "una vez levantada la declaración de emergencia sanitaria”; en el caso del artículo 2 del Decreto 45/2020 del Presidente de la Xunta de Galicia, de manera jurídicamente más torpe, se dispuso que la nueva convocatoria de elecciones se produciría "una vez levantada la declaración del estado de alarma y la situación de emergencia sanitaria". Es decir, en el caso gallego, siguiendo el tenor literal, se hacía depender la nueva convocatoria, no sólo de la mejoría de la situación sanitaria, sino también de la pérdida de vigencia del estado de alarma. Esta última cuestión hacía depender indirectamente la nueva convocatoria de elecciones en Galicia de la voluntad del Congreso de los Diputados, al ser la institución responsable de acordar las sucesivas prórrogas del estado de alarma, cosa que no parece justificada tratándose de elecciones de ámbito autonómico.

Esa decisión del Presidente de la Xunta de ligar la nueva convocatoria electoral al levantamiento del estado de alarma parece que fue poco reflexionada y, además, resulta constitucionalmente cuestionable. En cualquier caso, el Decreto 72/2020, de convocatoria de nuevas elecciones al Parlamento de Galicia, ignoró ese requisito, dado que cuando se convocaron de nuevo las elecciones autonómicas gallegas aún persistía la vigencia del estado de alarma. Este incumplimiento se hizo jurídicamente más tolerable si se tiene en cuenta que el Real Decreto 514/2020, de 8 de mayo, por el que se prorrogó el estado de alarma declarado por el Real Decreto 463/2020, de 14 de marzo, incluía una Disposición final primera que añadía un nuevo apartado 1 bis al artículo 7, del siguiente tenor: "la vigencia del estado de alarma no supondrá 
fundación

Manuel Giménez Abad

deEstudios Parlamentariosydel Estado Autonómico

obstáculo alguno al desenvolvimiento y realización de las actuaciones electorales precisas para el desarrollo de elecciones convocadas a Parlamentos de comunidades autónomas".

En los dos nuevos Decretos, además, se exigió que la nueva convocatoria de elecciones se efectuase "oídos los partidos políticos". Sin embargo, a diferencia del consenso amplio que se alcanzó entre los partidos a la hora de aceptar la cancelación de las elecciones, la nueva convocatoria no salió adelante con el visto bueno de todos ellos en ninguno de los dos casos (Cebrián, 2020: 31-33; Sánchez Navarro, 2020: 342). Asimismo, conviene aclarar que las distintas autoridades entendieron que no se trataba de reanudar los respectivos procesos electorales desde el momento en que se vieron interrumpidos, sino que estábamos ante auténticos nuevos procesos electorales que arrancaban desde el inicio, cosa que resultaba relevante, por ejemplo, a efectos de conformación del censo electoral o de la posibilidad de presentarse nuevos partidos, coaliciones o agrupaciones de electores (Arnaldo, 2020).

Para algunos autores, la decisión de suspender o anular las elecciones debe considerarse adecuada a la luz del principio democrático y del aseguramiento de la integridad en el proceso electoral, colmándose así una laguna jurídica en la normativa electoral española (Arnaldo, 2020; Cebrián, 2020; Sánchez Navarro, 2020; Riquelme, 2021). No en balde, existía una imposibilidad material de asegurar el correcto ejercicio de los derechos fundamentales de participación política, tanto en lo que respecta al derecho de sufragio activo (artículo 23.1 CE) como al de sufragio pasivo (artículo 23.2 CE). Mientras que, para otros autores, a los que me sumo, existen dudas sobre si los Decretos del Lehendakari y del Presidente de la Xunta de Galicia, por los que se dejaron sin efecto los procesos electorales que habían sido convocados, podrían haber supuesto una limitación constitucionalmente proscrita de los derechos de sufragio activo y pasivo (Barrat, 2021). Conviene recordar que el estado de alarma es uno de los tres estados de emergencia existentes en nuestro ordenamiento constitucional (artículo $116 \mathrm{CE}$ ), pero el único que no permite la suspensión generalizada de derechos fundamentales (artículo 55.1 CE). Por tanto, el estado de alarma tan solo permitiría la "limitación" de algunos de ellos, entre los que destaca la libertad de circulación del artículo 19 CE (artículo 11 a) de la Ley Orgánica 4/1981, de 1 de junio, de los estados de alarma, excepción y sitio -LOEAES-). De modo que, de un lado, el régimen constitucional de 
suspensión generalizada de los derechos fundamentales no prevé en ningún caso la suspensión de los derechos de sufragio reconocidos en el artículo 23 CE (artículo 55.1 CE); y, de otro lado, la declaración del estado de alarma no admite la suspensión de derechos fundamentales, solo la restricción de alguno de ellos, entre los que no se encuentra en ningún caso el derecho de sufragio. Desde esa óptica, pues, la decisión de suspender un proceso electoral ya convocado sin habilitación constitucional o legal de ningún tipo es, cuando menos, controvertida jurídicamente hablando.

Por lo demás, concuerdo con Miguel Presno (2020a) en que debería haberse dotado de cobertura legal a la cancelación electoral, por tratarse de una decisión que afecta a derechos fundamentales y al régimen electoral general (artículo 81.1 CE). A mi juicio, lo correcto habría sido modificar puntualmente la LOREG para permitir postergar unos comicios ya convocados en caso de darse circunstancias extraordinarias que imposibiliten el mantenimiento de la normalidad o la integridad del proceso electoral. Ese precepto bien podría ser un artículo 42 bis LOREG, que debería contemplar, al menos, las siguientes cuestiones: el tipo de situaciones excepcionales que habilitarían para tomar la decisión de cancelar el proceso electoral; la identificación de la autoridad competente para cancelar el proceso electoral ya iniciado; el procedimiento que habría de seguirse para proceder a la cancelación electoral; los plazos o situaciones que permitirían volver a convocar las elecciones desde un inicio y el procedimiento a seguir; y, por último, las garantías y posibles recursos que cabría interponer frente a un procedimiento tan políticamente delicado y tan democráticamente relevante. A su vez, ese artículo 42 bis LOREG requeriría su inclusión en la extensa lista de preceptos del apartado segundo de la Disposición adicional primera de la LOREG, para adquirir carácter básico y resultar aplicable a las comunidades autónomas.

Debe tenerse en cuenta que esta no es una posibilidad completamente ajena a nuestro ordenamiento jurídico. Así, como bien ha recordado Enrique Cebrián (2020: 16), el artículo 4.1 de la Ley Orgánica 2/1980, sobre regulación de las distintas modalidades de referéndum, sí contempla la suspensión de la celebración de referéndums ya convocados en caso de que se declaren los estados de excepción y de sitio: "No podrá celebrarse referéndum en ninguna de sus modalidades durante la vigencia de los estados de excepción y sitio en alguno de los ámbitos territoriales en los que se realiza la consulta o en los 
noventa días posteriores a su levantamiento. Si en la fecha de la declaración de dichos estados estuviere convocado un referéndum, quedará suspendida su celebración, que deberá ser objeto de nueva convocatoria”. A propósito, el hecho de que se establezca expresamente esta posibilidad en una ley orgánica aprobada en el año 1980, refuerza la idea de que, para llevar a cabo una suspensión similar de unas elecciones, debería contemplarse de modo semejante en alguna ley orgánica como la LOEAES, de 1981, o, más propiamente, en la LOREG, de 1985.

Lo cierto es que la controversia jurídica respecto a las elecciones en Galicia y País Vasco no se agotó ahí. En ambos comicios autonómicos se produjo también polémica con relación a los derechos de sufragio de los infectados por covid. Y es que, a unos pocos días de la jornada electoral, las autoridades gallegas y vascas decidieron no permitir que centenares de electores que habían dado positivo y a los que se les había impuesto una cuarentena de varios días pudiesen salir de su casa para acudir a los colegios electorales y ejercer su derecho al voto. Los organismos electorales avalaron en todos los casos las decisiones que impidieron votar a los enfermos por covid-19 en esas elecciones gallegas y vascas. Buena prueba de ello fueron los Acuerdos de la Junta Electoral del País Vasco 2020/357, 2020/360 y 2020/370, la Resolución de la Junta Electoral de Galicia de 7 de julio de 2020, y los Acuerdos de la Junta Electoral Central (JEC) 126/2020, 127/2020, 128/2020 y 129/2020 y su Resolución de 11 de julio de 2020. En los tres primeros acuerdos de la JEC, sin ir más lejos, el organismo electoral consideró que las medidas adoptadas por la Administración garantizaban "unas condiciones adecuadas de circulación para poder acudir a votar, con la salvedad de segmentos reducidos de población, por motivos sanitarios suficientemente justificados en el marco de la situación de pandemia en la que se están desarrollando las elecciones".

Por poner un ejemplo, se produjeron declaraciones explícitas de la Consejera de Seguridad del gobierno vasco, en las que ésta advirtió a las personas que se encontraban en dicha situación de las repercusiones que en forma de sanciones podría acarrear su desplazamiento a los colegios electorales si trataban de ejercer su derecho al voto. Este tipo de decisiones fue convalidado por las juntas electorales autonómicas y, en última instancia, por la JEC. Así, en su Acuerdo 127/2020, de 9 de julio, la JEC desestimó el recurso interpuesto por la coalición electoral Galicia en Común-Anova Mareas contra el Acuerdo de 
la Junta Electoral de Galicia, de 7 de julio de 2020, en relación con la adopción de medidas para asegurar el desarrollo de la jornada electoral en la comarca lucense de La Mariña y en toda Galicia. Frente a ese Acuerdo, se interpuso un recurso contencioso-administrativo en el que se solicitaba "la adopción de todas las medidas cautelarísimas legalmente oportunas para garantizar que todas las vecinas y vecinos de la comarca de A Mariña actualmente confinados puedan ejercer, democráticamente y con todas las garantías sanitarias, su derecho fundamental al voto en las elecciones al Parlamento de Galicia del próximo 12 de julio, garantizando al mismo tiempo que el proceso electoral se desarrolla con total transparencia, objetividad y respeto al principio de igualdad". Sin embargo, la Sala de lo Contencioso-Administrativo del Tribunal Supremo acordó en un Auto 11 de julio de 2020 desestimar la medida cautelarísima solicitada.

Se trata, a mi modo de ver, de decisiones jurídicamente muy cuestionables. Y ello por más que se persiguiese el objetivo legítimo de evitar la propagación del virus y, por tanto, se estuviese intentando proteger los derechos de los ciudadanos a la vida y a la integridad física (artículo $15 \mathrm{CE}$ ). Como ha señalado Miguel Presno (2020b), resulta muy discutible que las medidas respetasen el principio de proporcionalidad en las limitaciones a un derecho fundamental, en la medida en que cabía imaginar restricciones alternativas menos lesivas para el derecho de sufragio activo que la simple privación del derecho al voto de las personas contagiadas o en cuarentena. Como acertadamente ha subrayado Enrique Cebrián (2020: 35), la reforma de la LOREG introducida por la Ley Orgánica 2/2018, de 5 de diciembre, para garantizar el derecho de sufragio de las personas con discapacidad, introdujo un nuevo apartado segundo al artículo 3 de la LOREG muy relevante a estos efectos: "toda persona podrá ejercer su derecho de sufragio activo, consciente, libre y voluntariamente, cualquiera que sea su forma de comunicarlo y con los medios de apoyo que requiera". ¿Hasta qué punto el legislador orgánico no ha introducido con esta reforma un mandato a los poderes públicos para garantizar el derecho de sufragio activo de los ciudadanos en todas las circunstancias, esto es, con los medios de apoyo que requieran? A la luz de este precepto, del que incluso podría decirse que transforma parcialmente la naturaleza del derecho de sufragio para introducir en él una nueva dimensión prestacional, resulta difícil no ver una vulneración del citado derecho en las limitaciones introducidas por las autoridades. 
Además, en una primera aproximación comparada, es posible afirmar que estas decisiones contrastan con lo observado en otros países. Sería el ejemplo de Corea del Sur, donde se arbitraron medidas para garantizar que los pacientes con coronavirus pudiesen votar desde sus hogares o desde los hospitales en las elecciones legislativas (Spinelli, 2020). Otro ejemplo sería el de Singapur, donde los aspirantes a candidatos que no pudiesen presentar la documentación necesaria para su nominación, debido a una orden de cuarentena o a estar hospitalizados o enfermos, pudieron hacerlo a través de la autorización a un representante mediante poder notarial; y donde también se habilitaron instalaciones específicas para que los electores confinados en sus domicilios por estar infectados pudieran ejercer su derecho al voto. Es decir, se tomaron medidas destinadas a favorecer los derechos de sufragio activo y pasivo de sus ciudadanos. Por lo que respecta a Croacia, que celebró sus elecciones parlamentarias en julio de 2020, la autoridad electoral prohibió en un primer momento la participación en las elecciones de los pacientes confinados con coronavirus, pero el Tribunal Constitucional croata revocó la prohibición, permitiendo que los enfermos pudiesen votar mediante poder notarial. $O$, en fin, en Italia, donde las autoridades arbitraron un sistema de voto para garantizar a los ciudadanos infectados por covid-19, ubicados en un hospital o en cuarentena en su propio domicilio, la participación en las elecciones regionales y en el referéndum de reforma constitucional celebrados los días 20 y 21 de septiembre. Estos ejemplos comparados no hacen sino evidenciar que la respuesta ofrecida por las autoridades españolas pudo ser mucho más garantista con los derechos de participación política de los ciudadanos.

\section{LAS MEDIDAS EXCEPCIONALES ADOPTADAS PARA LA CELEBRACIÓN DE LAS ELECCIONES DURANTE LA PANDEMIA}

Aunque, como acabamos de ver, muchos países y regiones han suspendido o aplazado sus elecciones, también encontramos ejemplos de otros que han mantenido la fecha prevista inicialmente para la celebración de los comicios o que, tras su retraso, han acabado celebrándolos unos meses después. Según los datos que ofrece International IDEA (2020a), entre el 21 de febrero y el 13 de diciembre de 2020, al menos 99 países y territorios han celebrado elecciones, 51 de los cuales lo hicieron continuando con la fecha planeada originalmente para celebrar elecciones nacionales o subnacionales, a pesar de 
las preocupaciones relacionadas con la covid-19. Por mencionar algunos casos, podemos destacar las elecciones parlamentarias en Irán, Corea del Sur, Sri Lanka o Croacia, las elecciones generales en Israel, Eslovaquia o Surinam, o las elecciones presidenciales en Islandia o Burundi.

¿Qué tipo de medidas especiales y de prevención se están adoptando en estas elecciones? ¿Se están reformando las legislaciones electorales a pocos meses de las elecciones? Esta última cuestión no es baladí, pues estas reformas electorales reactivas o sobrevenidas, aprobadas a escasos meses de las elecciones, pueden representar una amenaza para la seguridad jurídica de los procesos electorales, amén de contravenir el Código de Buenas Prácticas en Materia Electoral elaborado por la Comisión de Venecia (2002), según el cual las reglas electorales no deben ser modificadas a menos de un año de la celebración de las elecciones. Si bien es cierto que la propia Comisión de Venecia (2020: 110), en su Compilación de opiniones e informes sobre estados de emergencia, afirma que hacer una reforma electoral en las modalidades de votación menos de un año antes de las elecciones puede no contravenir el Código de Buenas Prácticas si contribuye a unas elecciones justas.

Por lo que respecta a las decisiones adoptadas que afectan al proceso electoral, cabe destacar el acortamiento del período de campaña electoral, la cancelación de mítines y actos de campaña o la limitación del número de personas que podían asistir a los mismos. Estas campañas restringidas están provocando que en muchos casos la atención gire casi exclusivamente en torno a la crisis de salud pública, orillando otros temas relevantes e impidiendo una discusión más rica. Además, parece que las restricciones que la pandemia está obligando a desplegar con relación a los actos electorales están provocando una aceleración de transformaciones ya en curso, como una mayor presencia de partidos políticos y candidatos en los medios de comunicación y un creciente uso por parte de estos últimos de las redes sociales con el fin de atraer el voto.

Destacan asimismo las medidas adoptadas para proteger la salud de los participantes (Krimmer et al., 2020). Durante la jornada de votación, puede mencionarse el uso obligatorio de mascarillas para ciudadanos y funcionarios electorales; el control de temperatura a la entrada de los colegios electorales y el mantenimiento de la distancia social en su interior; la adopción de medidas higiénicas tales como la provisión de agua y jabón y geles desinfectantes a los 
accesos a los edificios; o la obligación de que los electores porten su propio bolígrafo para poder confeccionar su voto. En algunos casos, el recuento de los votos se ha retransmitido en directo en los medios de comunicación y a través de redes sociales, para respetar las restricciones y mantener el distanciamiento social sin que ello supusiese una merma en la publicidad del acto de escrutinio.

Ahora bien, a pesar de las medidas adoptadas ¿qué implicaciones ha podido tener la celebración de las elecciones en un contexto de pandemia? Una de las consecuencias podría haberse proyectado sobre los niveles de participación electoral. Uno de los primeros trabajos que ha analizado esta cuestión en las elecciones de todo el mundo celebradas en los primeros siete meses desde la irrupción de la pandemia mundial arroja resultados interesantes: mientras que la participación no habría descendido de forma generalizada en comparación con las elecciones celebradas en cada caso con anterioridad a la pandemia, sí se detecta un descenso en aquellos países y regiones donde la pandemia está golpeando de manera más severa, tanto en términos de infectados como, sobre todo, de fallecimientos (Santana, Rama y Casal-Bértoa, 2020). Además, ese descenso de la participación podría haberse manifestado especialmente en los grupos de electores vulnerables, como las personas mayores. De confirmarse esta expectativa, podrían verse comprometidos principios elementales que deben presidir los procesos electorales, como son los de participación, inclusión e igualdad.

Mención aparte merece todo lo relativo a las novedades asociadas a las distintas modalidades de votación empleadas. En bastantes casos, se ha apostado por "votaciones tempranas" (early voting) para que los ciudadanos pudiesen ejercer su derecho de sufragio durante varios días, lo que obviamente permite cumplir más fácilmente con el distanciamiento social. Con el mismo objetivo de evitar aglomeraciones en los centros de votación, en otros casos también se han establecido ventanas o franjas de votación escalonadas a lo largo de la jornada electoral.

Asimismo, se ha apostado por fórmulas alternativas de votación remota que, frente al voto presencial ordinario, reducirían los riesgos para la salud pública y favorecerían la participación de grupos vulnerables. Con carácter general, el voto por correo se ha revelado como una modalidad de votación clave en este específico contexto, fomentándose su utilización por parte de las autoridades electorales. Incluso se encuentran casos de elecciones celebradas 
exclusivamente en la modalidad de voto postal, como sucedió en las elecciones locales de Baviera. Ese iba a ser también el caso de las elecciones presidenciales de Polonia, donde en un primer momento el gobierno anunció su intención de que la única modalidad de votación fuese postal; sin embargo, ante las duras críticas recibidas, acabó buscando fórmulas de compromiso y la legislación aprobada finalmente concedió a los electores la opción de solicitar el voto por correo o emitirlo presencialmente en los colegios electorales (Vashchanka, 2020).

En España, en las elecciones autonómicas de Galicia y País Vasco, la JEC, en su Acuerdo 56/2020, de 28 de mayo, modificó algunas cuestiones procedimentales con el fin de facilitar los trámites para ejercer el voto por correo. Entre otras cosas, la JEC permitió que aquellos electores que lo prefiriesen pudiesen realizar la petición de forma telemática en la web de Correos, mediante firma electrónica; también que los electores no tuviesen por qué firmar personalmente la entrega, permitiéndoles demostrar su identidad mediante el DNI $u$ otro documento oficial; o que pudiesen recibir la documentación electoral y a continuación emitir su voto, sin necesidad de tener que acudir posteriormente a una oficina de Correos. Aunque no cabe duda de que la actitud de la JEC fue loable, lo cierto es que actuó más allá de sus competencias, acordando medidas que contradijeron abiertamente lo establecido en distintos apartados de los artículos 72 y 73 LOREG, comprometiendo incluso principios como los de personalidad y secreto del voto y, por tanto, sembraron la duda sobre su validez jurídica (Cebrián, 2020: 32-33; Barrat, 2021).

España no es el único lugar donde las autoridades han tratado de facilitar el voto por correo, tal y como han puesto de manifiesto las recientes elecciones en Estados Unidos. En Nueva Zelanda, en las elecciones generales pospuestas a octubre de 2020, la Comisión Electoral consideró la posibilidad de extender a todos los electores las formas de votación alternativas existentes, entre las que se encuentra el voto por correo, diseñadas originalmente para aquellos electores que no pueden asistir presencialmente a un colegio electoral como los electores en el extranjero o aquellos que tienen algún tipo de discapacidad física.

En todo caso, hay que tener en cuenta los problemas que suscita esa modalidad de votación. Para que unas elecciones puedan llevarse a cabo 
apoyándose en el voto por correo de forma generalizada, resulta imprescindible contar con un servicio postal eficaz, convenientemente dotado y bien instruido, que pueda afrontar con solvencia la carga de trabajo. Además, los costes económicos de sufragar ese tipo de votación son considerablemente mayores a los de una votación tradicional, debido al importante esfuerzo logístico e informativo que debe desplegarse, circunstancia que no todos los países o regiones están en disposición de afrontar. Por último, no siempre se dispone de tiempo suficiente para llevar a cabo una política de ese calado, y los plazos necesarios para capacitar a los empleados de los servicios postales o reclutar y adiestrar a nuevo personal pueden impedir la viabilidad de su implementación. Si no se cumplen esas exigencias, la decisión de apostar por la utilización masiva del voto por correo puede resultar un fracaso. Se corre el riesgo de que aumenten los episodios de coacciones y compra de votos que pondrían en riesgo la pureza de los sufragios, lo que generaría desconfianza entre los ciudadanos y, con ello, se debilitaría la legitimidad de los resultados y la integridad del proceso electoral.

La pandemia también ha servido para reavivar los debates sobre la tecnología aplicada a las elecciones y sobre el voto electrónico (Krimmer et al., 2020). Desde hace aproximadamente dos décadas, se ha desarrollado una importante literatura académica al respecto (Barrat y Fernández Riveira, 2011; Barrat, 2016; Gibson et al., 2016; Guglielmi e Ihl, 2017). Por lo que respecta al voto por internet o telemático, los argumentos a favor consisten en afirmar que esta modalidad de votación reduciría el coste de las elecciones, agilizaría los trámites, las volvería más accesibles y, por tanto, podrían verse aumentadas las tasas de participación. Sin embargo, la mayoría de países han evitado la votación telemática a gran escala al existir riesgos para la confianza, certidumbre y seguridad de las elecciones: posibles violaciones del secreto de sufragio; problemas en el correcto escrutinio de los votos emitidos; una inadecuada comprensión de los electores del sistema de votación; dificultades para que los observadores electorales puedan verificar el correcto desarrollo del proceso; o, en fin, los posibles ataques informáticos que puedan interferir en la votación o adulterar los resultados. Hasta el momento, su uso se ha limitado al relevante caso de Estonia y, en menor medida, a países que lo han empleado en alguna ocasión, para algún tipo de elecciones, como Canadá, Australia, Suiza o Noruega. 
Sin embargo, como forma de responder al desafío que plantea la crisis del covid-19, los países pueden considerar apostar por el voto por internet, que no deja de ser el equivalente telemático del voto por correo. De hecho, hay quien subraya las ventajas de impulsar el voto telemático frente al voto por correo: evitar la mayoría de los problemas logísticos de la votación postal, como el envío por correo de cientos de miles de sobres y papeletas; escrutinios más rápidos que los que consisten en el recuento manual de las papeletas y el aseguramiento de que los votos no llegan tarde al recuento; conjurar el riesgo de que los votos se extravíen en países donde los servicios postales no funcionan adecuadamente; reducir los votos nulos derivados de los errores formales cometidos por los electores al efectuar los trámites postales; o el ahorro financiero que podría suponer eliminar los costosos gastos relacionados con el envío generalizado y masivo de sobres y papeletas (Wolf, 2020). Lo cierto es que aún desconocemos cuánto durará la crisis, pero, cuanto más dilatada sea, más probable será que observemos desarrollos en esta dirección.

\section{UNA NUEVA AGENDA DE INVESTIGACIÓN}

En este trabajo he tratado de proporcionar una primera aproximación al impacto que está teniendo la pandemia mundial del covid-19 en la organización y desarrollo de los procesos electorales. Es decir, he intentado mostrar una panorámica de cuáles son las principales respuestas que las autoridades están dando. Mientras que en algunos países y regiones se han cancelado o aplazado las elecciones, en otros lugares se ha decidido seguir adelante adoptando medidas excepcionales. He prestado especial atención al caso autonómico español. En las elecciones de Galicia y de País Vasco, celebradas en 2020, las distintas autoridades adoptaron decisiones jurídicamente controvertidas tanto en lo que se refiere al modo en que se procedió a cancelar y convocar de nuevo los procesos electorales, como en las medidas adoptadas para facilitar el voto por correo. Con todo, lo más criticable de esas actuaciones, como he argumentado, me parece la restricción del derecho de sufragio de las personas contagiadas por covid-19 o en cuarentena.

En cualquier caso, esta aproximación preliminar pone de manifiesto la necesidad de profundizar en el estudio sistemático de todas estas cuestiones en perspectiva comparada. ¿Cómo está afectando la pandemia a la 
organización y desarrollo de los procesos electorales en todo el mundo? ¿Qué decisiones están adoptando las autoridades para poder celebrar las elecciones y garantizar el derecho de sufragio de los ciudadanos? ¿Qué instrumentos jurídicos se están empleando para adoptar esas medidas extraordinarias? Y ¿cuál es su cobertura constitucional o legal?

Los investigadores de las cuestiones electorales debemos tratar de responder algunas cuestiones relativas al aplazamiento de las elecciones o a la adopción de medidas especiales en aquellos casos en los que se decide seguir adelante con su celebración. Por ejemplo, respecto al aplazamiento de las elecciones motivadas por la pandemia ¿qué instrumentos jurídicos se están empleando para suspender o posponer las elecciones? ¿Existe cobertura constitucional o legal para ello? ¿Los aplazamientos de las elecciones está siendo presididos por los acuerdos entre los partidos o se están imponiendo de forma unilateral por los gobernantes? ¿Está siendo aprovechada esta situación por parte de los partidos en el poder para extender su mandato, laminar a los partidos de la oposición u obtener ventajas electorales? ¿Cómo están afectando los aplazamientos electorales a la organización y funcionamiento de los distintos Parlamentos? ¿Cómo está incidiendo esa eventualidad en el mandato de los parlamentarios? ¿Convendría llevar a cabo una reforma de la LOREG para desarrollar un régimen jurídico específico aplicable a los aplazamientos electorales?

Y respecto a las medidas adoptadas para la celebración de las elecciones durante la pandemia ¿qué tipo de medidas están acordando las autoridades para garantizar la seguridad sanitaria y el correcto desarrollo de los procesos electorales? ¿Qué respuestas están ofreciendo con relación al derecho de sufragio de las personas infectadas por covid? ¿Se están reformando las legislaciones electorales para introducir mecanismos que ajusten la normativa a la situación de pandemia actual? ¿Las distintas restricciones relacionadas con las campañas electorales están afectando a la calidad del debate público? ¿Esas restricciones a la actividad de captación de sufragios por parte de los partidos están situando en condiciones de ventaja competitiva a los que se encuentran en el poder frente a los de la oposición o a eventuales nuevas opciones políticas? ¿Se percibe un incremento significativo del uso de internet y de las redes sociales por parte de candidatos y partidos políticos para atraer el voto? ¿Ha aumentado la abstención debido al contexto de pandemia en el 
que se han celebrado las elecciones? ¿Se ha deprimido la participación de forma asimétrica, incidiendo en los electores más propensos a padecer la enfermedad de forma grave? ¿Están fomentando las autoridades la utilización de fórmulas alternativas de votación como el voto por correo? ¿Están siendo sus rendimientos satisfactorios? ¿Se observan avances en la utilización del voto telemático para las elecciones o dicha modalidad de votación sigue suscitando recelos entre las autoridades?

Ofrecer una respuesta a estas y otras preguntas sin duda reforzaría lo que Arend Lijphart (1997) denominase hace tiempo como la ciencia de los sistemas electorales, esto es, un conocimiento sistemático y riguroso sobre la adopción, el funcionamiento o las consecuencias de ese conjunto sustancial de las reglas que disciplinan el juego electoral democrático. A mi modo de ver, esa ciencia ha experimentado progresos considerables en las últimas décadas en lo que se refiere al conocimiento de los sistemas electorales en sentido estricto (Herron, Pekkanen y Shugart, 2018). También ha avanzado notablemente la investigación internacional sobre la organización e integridad de los procesos electorales (Massicotte, Blais y Yoshinaka, 2004; Catt et al., 2014; Norris, 2014; James, 2020b). Pero se encuentra todavía subdesarrollada en lo que se refiere al impacto que desastres naturales o una pandemia de impacto global como la actual pueden tener en todo lo relativo a las elecciones. Constitucionalistas y politólogos tenemos un nuevo reto investigador apasionante por delante.

\section{BIBLIOGRAFÍA}

- ARNALDO, E. (2020). La suspensión de la celebración de las elecciones comporta una nueva convocatoria íntegra. Diario La Ley, 9608, 6 de abril de 2020.

- BARRAT, J. y FERNÁNDEZ RIVEIRA, R. M. (2011). Derecho de sufragio y participación ciudadana a través de las nuevas tecnologías. Madrid: Civitas.

- BARRAT, J. (2016). El voto electrónico y sus dimensiones jurídicas: entre la ingenua complacencia y el rechazo precipitado. Madrid: lustel. 
- (2021). Spanish Elections under Pandemic. Elections and COVID-19. Research from the Electoral Management Network \& International IDEA. [Forthcoming, work in progress].

- CATT, H.; ELLIS, A.; MALEY, M.; WALL, A., y WOLF, P. (2014). Electoral management design: Revised edition. International IDEA.

- CEBRIÁN, E. (2020). COVID-19 y anulación de procesos electorales autonómicos en País Vasco y Galicia. Revista General de Derecho Constitucional, 33.

- Comisión de Venecia (2002). Code of good practice in electoral matters. Opinion 190/2002. Disponible en: https://www.venice.coe.int/webforms/documents/default.\%20aspx?pd\%E F\%AC\%83le=CDL-AD(2002)023rev2-cor-e. [Consultado el 08/12/2020].

- (2020). Compilation of Venice Commission Opinions and Reports on States of Emergency. CDL-PI(2020)003. Disponible en: https://www.venice.coe.int/webforms/documents/?pdf=CDLPI(2020)003-e [Consultado el 08/12/2020].

- DAHL, R. (1971). Polyarchy: Participation and Opposition. New Haven: Yale University Press.

- GIBSON, J. P.; KRIMMER, R.; TEAGUE, V. y POMARES, J. (2016). A review of e-voting: the past, present and future. Annals of Telecommunications, 71 (7-8), 279-286.

- GUGLIELMI, G. e IHL, O. (2017). El voto electrónico. Madrid: Centro de Estudios Políticos y Constitucionales.

- HERRON, E. S.; PEKKANEN, R. J., y SHUGART, M. S. (Eds.) (2018). The Oxford Handbook of Electoral Systems. Oxford: Oxford University Press.

- International IDEA (2020a). Global overview of Covid -19: Impact on elections. Disponible en: https://www.idea.int/news-media/multimedia- 
reports/global-overview-covid-19-impact-elections [Consultado el 08/12/2020].

- (2020b). Elections and COVID-19. International IDEA Technical Paper 1/2020. Disponible en:

https://www.idea.int/sites/default/files/publications/elections-andcovid-19.pdf [Consultado el 08/12/2020].

- JAMES, T. S. (2020a). New development: Running elections during a pandemic. Public Money \& Management, 1-4.

- (2020b). Comparative electoral management: Performance, networks and instruments. Routledge.

- JAMES, T. S., y ALIHODZIC, S. (2020). "When is it democratic to postpone an election? Elections during natural disasters, Covid-19 and emergency situations", Election Law Journal, 19(3), pp. 344-362.

- KRIMMER, R.; DUENAS-CID, D., y KRIVONOSOVA, I. (2020). Debate: safeguarding democracy during pandemics. Social distancing, postal, or internet voting-the good, the bad or the ugly? Public Money \& Management, 1-3.

- LIJPHART, A. (1997). The difficult science of electoral systems: A commentary on the critique by Alberto Penadés. Electoral Studies, 16(1), 73-77.

- MASSICOTTE, L.; BLAIS, A., y YOSHINAKA, A. (2004). Establishing the rules of the game: Election laws in democracies. University of Toronto Press.

- NORRIS, P. (2014). Why electoral integrity matters. Cambridge University Press.

- PRESNO LINERA, M. Á. (2020a). Coranavirus SARS-CoV-2 y derechos fundamentales (5): el derecho de voto (suspensión de elecciones vascas y gallegas). El derecho y el revés (3 de abril de 2020). Disponible en: https://presnolinera.wordpress.com/2020/04/03/coranavirus-sars-cov-2- 
$y$-derechos-fundamentales-5-el-derecho-de-voto-suspension-deelecciones-vascas-y-gallegas/ [Consultado el 08/12/2020].

- (2020b). Prohibido prohibir votar. Agenda Pública (12 de julio de 2020). Disponible en: http://agendapublica.elpais.com/prohibidoprohibir-votar/ [Consultado el 08/12/2020].

- RIQUELME, P. (2021). Elecciones en tiempos de pandemia: la experiencia de las Comunidades Autónomas de Galicia y el País Vasco. En A. Dueñas, D. Fernández, P. Guerrero y G. Moreno (coords.). La Constitución en tiempos de pandemia. Valladolid: Dykinson/Universidad de Valladolid.

- SPINELLI, A. (2020). Managing Elections under the COVID-19 Pandemic. The Republic of Korea's Crucial Test. International IDEA Technical Paper 2/2020. Disponible en:

https://www.idea.int/sites/default/files/publications/managing-electionsduring-pandemic-republic-korea-crucial-test.pdf [Consultado el 08/12/2020].

- SÁNCHEZ NAVARRO, Á. (2020). Emergencia sanitaria y suspensión de elecciones. En D. Barceló, S. Díaz, J. García y M. E. Guimaraes (coords.). COVID 19 y parlamentarismo. Los Parlamentos en cuarentena (pp. 331-344). Ciudad de México: Universidad Nacional Autónoma de México.

- SANTANA, A.; RAMA, J. y CASAL-BÉRTOA, F. (2020). The Coronavirus Pandemic and Voter Turnout: Addressing the Impact of Covid on Electoral Participation. [Forthcoming, work in progress].

- VASHCHANKA, V. (2020). Political manoeuvres and legal conundrums amid the COVID-19 pandemic: the 2020 presidential election in Poland. International IDEA. Disponible en:

https://www.idea.int/sites/default/files/political-manoeuvres-and-legalconundrums-2020-presidential-election-poland.pdf [Consultado el 08/12/2020]. 
fundación

Manuel Giménez Abad

deEstudios Parlamentariosydel Estado Autonómico

- WOLF, P. (2020). The COVID-19 crisis - A much needed new opportunity for online voting?. International IDEA. Disponible en: https://www.idea.int/news-media/news/covid-19-crisis-\%E2\%80\%93much-needed-new-opportunity-online-voting [Consultado el 08/12/2020]. 